\title{
Intrinsically disordered nuclear pore proteins show ideal-polymer morphologies and dynamics
}

\author{
Luke K. Davis $\odot,{ }^{1,2,3}$ Ian J. Ford, ${ }^{1,2}$ Anđela Šarić $\odot,{ }^{2,3, *}$ and Bart W. Hoogenboom ${ }^{1,2,3, \dagger}$ \\ ${ }^{1}$ London Centre for Nanotechnology, University College London, London WC1H OAH, United Kingdom \\ ${ }^{2}$ Department of Physics and Astronomy, University College London, London WC1E 6BT, United Kingdom \\ ${ }^{3}$ Institute for the Physics of Living Systems, University College London, London WC1E 6BT, United Kingdom
}

(Received 15 June 2019; accepted 3 February 2020; published 28 February 2020)

\begin{abstract}
In the nuclear pore complex, intrinsically disordered nuclear pore proteins (FG Nups) form a selective barrier for transport into and out of the cell nucleus, in a way that remains poorly understood. The collective FG Nup behavior has long been conceptualized either as a polymer brush, dominated by entropic and excluded-volume (repulsive) interactions, or as a hydrogel, dominated by cohesive (attractive) interactions between FG Nups. Here we compare mesoscale computational simulations with a wide range of experimental data to demonstrate that FG Nups are at the crossover point between these two regimes. Specifically, we find that repulsive and attractive interactions are balanced, resulting in morphologies and dynamics that are close to those of ideal polymer chains. We demonstrate that this property of FG Nups yields sufficient cohesion to seal the transport barrier, and yet maintains fast dynamics at the molecular scale, permitting the rapid polymer rearrangements needed for transport events.
\end{abstract}

DOI: 10.1103/PhysRevE.101.022420

\section{INTRODUCTION}

Nuclear pore complexes (NPCs) penetrate the nuclear envelope in eukaryotic cells, controlling macromolecular transport between the nucleus and cytoplasm. The NPC enables small $(\lesssim 5 \mathrm{~nm}$ in size) molecules to cross the nuclear envelope, but hinders the transport of larger macromolecules [1-3]. Larger macromolecular cargos, however, can diffuse through the NPC if they are bound to nuclear transport receptors that have an affinity to the transport barrier. Remarkably, the NPC maintains the transport barrier while thousands of cargos shuttle in and out of the cell nucleus per second [4]. This transport barrier consists of proteins (nucleoporins) that are rich in phenylalanine $(\mathrm{F})$ and glycine $(\mathrm{G})$ repeats (hence called FG Nups, for FG nucleoporins) and that are grafted to the inner wall of the NPC transport channel. These hydrophobic domains grant the FG Nups cohesive properties, which may be counterbalanced by electrostatic interactions $[5,6]$. FG Nups are intrinsically disordered proteins [7], causing them to behave as flexible polymers [8].

For a long time, there have been conflicting views on the dominant interactions pervading FG Nup assemblies, and these views continue to define the interpretation of experimental data. On one hand, repulsive interactions have been postulated to result in entropic polymer-brush behavior of FG Nups [9-11]. On the other hand, cohesive (i.e., attractive) interactions can cause FG Nups to form hydrogels in vitro $[4,12,13]$. The combined effects of repulsion, cohesion, grafting, and nanopore confinement lead to a rich landscape of possible polymer behavior [14-17].

\footnotetext{
*a.saric@ucl.ac.uk

†b.hoogenboom@ucl.ac.uk
}

Recently, more nuanced views regarding FG Nup interactions have emerged, substantiated by computational models of FG Nups at different levels of coarse graining. By defining FG Nups down to their specific amino acid composition, such models can relate, e.g., FG Nup behavior to their chemical composition and can explore the effects of mutations [18-20], with the important caveat that the results critically depend on a large number of parameters describing the various sizes, charges, and hydrophobicities of the amino acids. Complementarily, at a coarser ("mesoscale") level, FG Nups have been modeled as homogeneous polymers where the electrostatic, hydrophobic, and hydrophilic interactions are incorporated into one essential interaction parameter [21,22]. Remarkably, these coarser models have reproduced key functional properties of FG Nups, with and without nuclear transport receptors, as observed in experiment $[21,22]$. This strongly suggests that NPC transport functionality may be generically understood in terms of mesoscale polymer physics. These and other studies have also indicated that FG Nup assemblies display aspects of both entropic and cohesive physical behavior $[16,21,22]$. Furthermore, there is experimental evidence that isolated intrinsically disordered proteins, including FG Nups, show properties that are close to those of "ideal" polymers $[23,24]$, characterized by a radius of gyration scaling of $R_{G} \propto N^{1 / 2}$, with $N$ being the number of monomers, where repulsive and attractive interactions balance such that the polymer behaves as if no excluded-volume or other larger-range intramolecular interactions are present [25].

In this paper, we show how such ideal-polymer behavior compares with experimental data on 27 purified FG domains that are either isolated or grafted at physiological densities (FG Nup assemblies) $[5,10,22,26,27]$. Specifically, we assess how the experimental morphologies compare with computational predictions on polymer behavior from assemblies containing repulsive, cohesive, and ideal polymers. Importantly, 
we also investigate how these behaviors translate into the static and dynamic properties of polymers in a context that is relevant to NPC transport functionality.

\section{METHODS}

\section{A. Molecular dynamics (MD)}

In order to investigate polymer morphology and dynamics, we have utilized MD where, following previous work $[15,22,28]$, FG Nups were modeled as polymers consisting of $N$ identical beads with diameter $d$ and bond length $r_{0}$, both set to $0.76 \mathrm{~nm}$. This choice in polymer model yielded a predicted persistence length of the approximate size of one amino acid $(0.38 \mathrm{~nm})$, which agrees with experimental data on FG Nups and other disordered polypeptide chains [29,30]. In addition, this choice results in a total excluded volume that approaches the excluded volume of an FG Nup (i.e., the sum of the van der Waals volumes of its amino acids [31], overestimating it by $\sim 20 \%$ on average; see Table S1 of the Supplemental Material [56]). Bonds were implemented using a harmonic potential, $U_{\text {bond }}(r)=\frac{1}{2} k\left(r-r_{0}\right)^{2}$, with a spring constant $k=500 k_{B} T / \mathrm{nm}^{2}$, where $r$ is the distance between the centers of two beads. To capture excluded-volume effects between amino acids and the cohesion arising from attractive - mainly hydrophobic — interactions, we imposed a combined (piecewise) pair potential between polymer beads [32] in which excluded-volume and cohesive interactions were modeled by separate pair potentials so that they can be changed independently. The excluded-volume interaction is the Weeks-Chandler-Andersen (WCA) potential given by

$$
U_{\mathrm{vol}}(r)= \begin{cases}4 \epsilon_{L J}\left[\left(\frac{\sigma}{r}\right)^{12}-\left(\frac{\sigma}{r}\right)^{6}\right]+\epsilon_{L J}, & r \leqslant d \\ 0, & d<r,\end{cases}
$$

where $\epsilon_{L J}=500 k_{B} T$ is the interaction strength and $\sigma=$ $2^{-\frac{1}{6}} d$; the addition of $\epsilon_{L J}$ to the potential ensured that $U_{\mathrm{vol}}(r=$ $d)=0.0 k_{B} T$. The cohesive interaction is based on an infinitely ranged attractive pair potential,

$$
U(r)= \begin{cases}0, & r<d \\ -\epsilon_{p p} \exp \left(\frac{d-r}{\lambda}\right), & d \leqslant r,\end{cases}
$$

where $\epsilon_{p p}$ is the cohesion strength and $\lambda$ is the decay length [15]. We set $\lambda=0.76 \mathrm{~nm}$ and we imposed that no two beads interacted beyond the cutoff distance $r_{c}=1.52 \mathrm{~nm}$, as it is not possible to have an infinitely ranged potential in MD. In order to ensure the continuity of the pair potential at $r_{c}$, we truncated and shifted the potential given by Eq. (2) using $U_{\text {att }}(r)=U(r)-U\left(r=r_{c}\right)-\left(\frac{d U(r)}{d r}\right)_{r=r_{c}}\left(r-r_{c}\right)$ [33], where $U_{\text {att }}(r)$ is the resulting cohesive pair potential given by

$$
U_{\text {att }}(r)=-\frac{\epsilon_{p p}}{A}\left\{\exp \left(1-\frac{r}{d}\right)\left[1-\frac{r\left(r-r_{c}\right)}{r d}\right]-\frac{1}{e}\right\},
$$

where $e$ is Euler's number and $A=1+(d / l)^{2}-1 / e$, where $l=1.0 \mathrm{~nm}$ is the unit of length. This ensured that the minimum of $U_{\text {att }}(r=d)=\epsilon_{p p}$. The total bead-bead pair potential, $U_{p p}(r)$, with well depth $\epsilon_{p p}$ is then given as

$$
U_{p p}(r)= \begin{cases}U_{\mathrm{vol}}(r)-\epsilon_{p p}, & r<d \\ U_{\mathrm{att}}(r), & d \leqslant r<r_{c} \\ 0, & r_{c} \leqslant r,\end{cases}
$$

where the minimum of $U_{\mathrm{vol}}(r)$ is brought down to $\epsilon_{p p}$ to ensure continuity at $r=d$ (Fig. S1(a) of the Supplemental Material [56]). MD simulations were performed using the LAMMPS package [34]. We subjected the polymer system to Langevin dynamics at a constant temperature $T$, by implementing the $N V E$ (constant number of beads, $N$, constant volume $V$, constant total energy $E$ ) time integration algorithm in combination with a Langevin thermostat. We performed the simulations with dimensionless parameters with $T=1$ and $\gamma=1$, where $\gamma$ is the friction coefficient, and a simulation time step of $\delta t=0.002$. To map simulation time to real time, we mapped one simulation time step to $3.4 \times 10^{-6} \mu$ s such that the self-diffusion time of one bead in our model matched the self-diffusion time of two attached amino acids (with size $\approx 0.76 \mathrm{~nm}$ ) in water at room temperature (see Supplemental Material [56]). At least $34 \mu \mathrm{s}$ were used to equilibrate the simulations, where equilibration was verified by inspection of the radius of gyration.

\section{B. Density functional theory (DFT)}

To investigate molecular interactions in a polymer system, we have used classical density functional theory (DFT), a scheme based upon the minimization of a dimensionless free energy functional $\mathcal{F}$ that depends solely on the number density of beads $\rho(r)$, and is written as $\mathcal{F}[\rho(r)]$ [35]. In this work, we have formulated DFT using mean-field theory so that the many-body polymer interactions are reduced to a single polymer interacting with a dimensionless mean field $w(r)$. The optimum mean field minimizes $\mathcal{F}$ and produces as output the equilibrium number density.

To model planar assemblies of FG Nups, we formulated a one-dimensional (1D) version of a previously successful 2D DFT formulation [22], where polymers were grafted onto the base of a cylinder with the assumption of rotational symmetry along the axial coordinate. This DFT has been previously described in extensive detail [15,22,28]. Here, we describe the $1 \mathrm{D}$ version, consisting of polymers grafted onto a flat surface and assuming translational symmetry along this surface. The determining coordinate was therefore the height $z$ above the grafting surface.

We took the approximation $\mathcal{F}=\mathcal{F}_{0}+\left\{\mathcal{F}_{\text {vol }}+\mathcal{F}_{\text {att }}+\right.$ $\left.\mathcal{F}_{\text {sur }}+\mathcal{F}_{\text {mf }}\right\}$, where $\mathcal{F}_{0}$ is the free energy functional describing a chain of $N$ noninteracting pointlike beads in the mean field, and excess terms representing excluded-volume, attractive, surface, and compensating mean-field interactions, respectively. $\mathcal{F}_{0}$ is defined by the Hamiltonian

$$
H_{0}=\sum_{i=0}^{N-1} h\left(r_{i+1}, r_{i}\right)+k_{B} T \sum_{i=1}^{N} w\left(z_{i}\right),
$$

where $h$ is a function (and $r$ is the magnitude of bead separation) that imposes a rigid bond length of $r_{0}$ between beads in a chain and $w(z)$ is the $1 \mathrm{D}$ mean field.

$\mathcal{F}_{\text {vol }}$ is the free energy functional imposing the excluded volume interactions between beads. To impose the excluded volume interactions, we used fundamental measure theory,

$$
\mathcal{F}_{\mathrm{vol}}=\int\left(\phi_{\mathrm{wb}}+\phi_{\mathrm{ch}}\right) d z
$$


where $\phi_{\mathrm{wb}}$ is the white-bear functional [36] and $\phi_{\mathrm{ch}}$ is the chain connectivity functional [37]. $\phi_{\mathrm{wb}}$ is given by

$$
\begin{aligned}
\phi_{\mathrm{wb}}= & -n_{0} \ln \left(1-n_{3}\right)+\frac{n_{1} n_{2}-\bar{n}_{1} \cdot \bar{n}_{2}}{1-n_{3}} \\
& +\left(n_{2}^{3}-3 n_{2} \bar{n}_{2}^{2}\right) \frac{n_{3}+\left(1-n_{3}\right)^{2} \ln \left(1-n_{3}\right)}{36 \pi n_{3}^{2}(1-n 3)^{2}},
\end{aligned}
$$

and $\phi_{\mathrm{ch}}$ is

$$
\begin{aligned}
\phi_{\mathrm{ch}}= & \left(\frac{1-N}{N}\right) n_{0}\left(1-\frac{\bar{n}_{2}^{2}}{n_{2}^{2}}\right) \\
& \times \ln \left[\frac{1}{1-n_{3}}+\frac{n_{2} R\left(1-\frac{\bar{n}_{2}^{2}}{n_{2}^{2}}\right)}{2\left(1-n_{3}\right)^{2}}+\frac{n_{2} R^{2}\left(1-\frac{\bar{n}_{2}^{2}}{n_{2}^{2}}\right)}{18\left(1-n_{3}\right)^{3}}\right],
\end{aligned}
$$

where $R=d / 2$ is the bead radius and $\left\{n_{\alpha}\right\}$ and $\left\{\bar{n}_{\alpha}\right\}$ are sets of scalar and vector weighted densities, respectively, that are given by

$$
\begin{aligned}
& n_{\alpha}(z)=\int \rho\left(z^{\prime}\right) \omega_{\alpha}\left(z-z^{\prime}\right) d z, \alpha=0,1,2,3, \\
& \bar{n}_{\alpha}(z)=\int \rho\left(z^{\prime}\right) \bar{\omega}_{\alpha}\left(z-z^{\prime}\right) d z, \alpha=1,2,
\end{aligned}
$$

where $\rho(z)$ is the one-dimensional number density, and $\omega_{\alpha}$ and $\bar{\omega}_{\alpha}$ are the 1D geometrical weight functions of a sphere [38] given as $w_{2}(z)=2 \pi R \theta(R-|z|), \bar{w}_{2}(z)=2 \pi z \bar{e}_{z} \theta(R-$ $|z|), \quad w_{3}(z)=\pi\left(R^{2}-z^{2}\right) \theta(R-|z|), \quad w_{1}(z)=w_{2}(z) /(4 \pi R)$, $\bar{w}_{1}(z)=\bar{w}_{2}(z) /(4 \pi R)$, and $w_{0}(z)=w_{2}(z) /\left(4 \pi R^{2}\right)$, where $\bar{e}_{z}$ is a unit vector and $\theta$ is the Heaviside function.

The cohesive term in the free energy, $\mathcal{F}_{\text {att }}$, is implemented using the random phase approximation [39] and is given by

$$
\mathcal{F}_{\text {att }}=\frac{\beta}{2} \iint \rho\left(z^{\prime}\right) \rho(z) U_{\text {att }}^{\perp}\left(z-z^{\prime}\right) d z d z^{\prime},
$$

where $\beta=1 / k_{B} T$ and $U_{\text {att }}^{\perp}(z)=\int_{-\infty}^{\infty} \int_{-\infty}^{\infty} U_{\text {att }}(r) d x d y$ (integrated over an infinite grafting area), with $r$ being the magnitude of vector separation between beads, and $U_{\text {att }}$ given by Eq. (3).

The free energy term representing the interactions between beads and the surface is given as

$$
\mathcal{F}_{\text {sur }}=\beta \int \rho(z) U_{\text {sur }}(z) d z
$$

with $U_{\text {sur }}$ as given in Eq. (1) in the Supplemental Material [56]. The mean-field energy $\mathcal{F}_{\mathrm{mf}}$ is the dimensionless free energy term that compensates for the introduction of a mean field and is given as

$$
\mathcal{F}_{\mathrm{mf}}=\int w(z) \rho(z) d z .
$$

To incorporate a number of polymers, $N_{p}$, one multiplies $\mathcal{F}_{0}$ by $N_{p}$ and interprets $\rho(z)$ as the number density of $N_{p} N$ beads. To compute $\rho(z)$, we solved the 1D diffusion equation for a random walk with contour length $N r_{0}$ in the presence of an external field $w(z)$ [15]. We optimized $w(z)$ through a discrete (a) Polymer Model

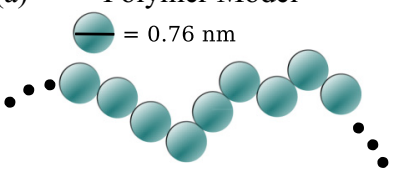

(b)
1 bead $\approx 2$ amino acids
Bead-bead interactions
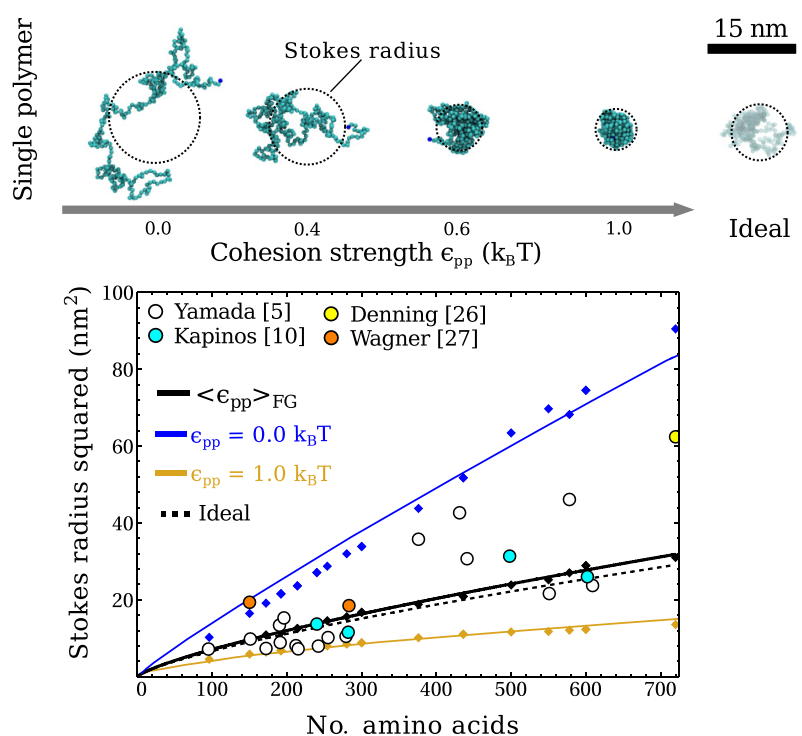

FIG. 1. Parametrization of the polymer model by comparison with experimental Stokes radii [5,10,26,27]. (a) Illustration of the polymer model (MD). (b) MD snapshots of a polymer $(N=300$ beads) as a function of $\epsilon_{p p}$, alongside an ideal polymer. (c) Experimental Stokes radii (circles) plotted against FG Nup sequence lengths (see Table S1 of the Supplemental Material [56]), compared with MD data (diamonds) alongside power law fits of the simulation data (solid and dashed lines).

update rule,

$$
\begin{aligned}
w_{n+1}\left(z_{j}\right)= & w_{n}\left(z_{j}\right)+\Delta t\left[-w_{n}\left(z_{j}\right)+\mu_{n}\left(z_{j}\right)\right. \\
& \left.+\beta \sum_{i}^{M} \rho_{n}\left(z_{i}\right) U_{\text {att }}^{\perp}\left(z_{i}-z_{j}\right) \Delta z+\beta U_{\text {sur }}\left(z_{j}\right)\right],
\end{aligned}
$$

where $n$ is an index representing the current iteration, $\Delta t$ is the update time step, $\{i, j\}$ are labels denoting discrete space, $\mu$ is the functional derivative of $\mathcal{F}_{\text {vol }}$ with respect to $\rho(z)$, and $M$ is the total number of discrete spatial points (along the $z$ axis). To ensure the stability of the update rule, we used $M=1024$ and $\Delta z=z_{\max } / M\left(z_{\max }=100 \mathrm{~nm}\right.$ so that polymer beads were well within the spatial domain), $\Delta t=0.001$, and the initial mean field was set to zero for all $z$. Convergence was obtained when $w_{n+1}\left(z_{j}\right)-w_{n}\left(z_{j}\right) \leqslant 10^{-7}$ for all $j$.

\section{RESULTS}

We model FG Nups as freely jointed polymers consisting of $N$ identical beads where one bead represents two amino acids [Fig. 1(a)]. FG Nup excluded-volume (repulsive) and cohesive interactions are modeled through a short-ranged pair potential [Eq. (4)] with a minimum value of $\epsilon_{p p}$, which is 
a measure of the cohesion strength between polymer beads. Thus defined, $\epsilon_{p p}$ is a phenomenological parameter capturing the general cohesive properties of FG Nups arising from the different attractive interactions between the amino acids. We consider weak attractive interactions, with a $K_{D} \sim 0.1 \mathrm{M}$ between two beads, as found here for $0.0 \leqslant \epsilon_{p p} \leqslant 1.0 k T$ [40] (see Fig. S1(b) of the Supplemental Material [56]).

Using an approach previously reported in the literature [41], the physiologically relevant $\epsilon_{p p}$ was determined by calculating hydrodynamic (Stokes) radii of specific FG constructs using MD simulations and the HYDRO++ program [42], and by comparing these with experimental data [5,10,26,27] (see Table S1 of the Supplemental Material [56] for the FG Nups used and the resulting $\epsilon_{p p}$ values).

Qualitatively, at $\epsilon_{p p}=0.0 k_{B} T$ (no cohesion), a polymer is in a swollen state; for $0.0<\epsilon_{p p}<1.0 k_{B} T$, polymers adopt morphologies that become increasingly compact with increasing $\epsilon_{p p}$; and at $\epsilon_{p p}=1.0 k_{B} T$ ("high cohesion"), a polymer forms a tight ball-like morphology (see Fig. 1(b), and Video S1 of the Supplemental Material [56]). When all bead-bead interactions are nullified (ideal polymer), the polymer sizes lie-as expected-between the predictions for polymers with no and high cohesion (with excluded volume).

The average of the parametrized $\epsilon_{p p}$ values (see Table S1 of the Supplemental Material [56]) yields $\left\langle\epsilon_{p p}\right\rangle_{F G}=0.5 \pm$ $0.2 k_{B} T$ [mean \pm standard deviation; see Fig. 1(c)]. Remarkably, the experimental data points for polymers with cohesion strength $\left\langle\epsilon_{p p}\right\rangle_{F G}$ coincide with the data from ideal polymer simulations [Fig. 1(c)]. This is a manifestation of polymer behavior that typically occurs at $\Theta$-point conditions [25]. Comparing the simulations for $\left\langle\epsilon_{p p}\right\rangle_{F G}$ with those for ideal polymers, we also find similar scaling exponents for Stokes radii (Table S2 of the Supplemental Material [56]) which are slightly less than the scaling exponents for $R_{G}$, as is known for finite length polymers [43].

Importantly, we observe that the match between the mean FG Nup behavior, with $\left\langle\epsilon_{p p}\right\rangle_{F G}$, and ideal polymer behavior is robust against the choice of model. Using a model (with a bead size of $0.57 \mathrm{~nm}$ ) with lower estimates of the protein volume and persistence length of FG Nups, we find a smaller $\left\langle\epsilon_{p p}\right\rangle_{F G}=0.26 \pm 0.06 k_{B} T$, yet for this $\left\langle\epsilon_{p p}\right\rangle_{F G}$, we still observe an excellent match with the ideal polymer prediction (Fig. S2 of the Supplemental Material [56]). Generally, to match the experimental data, the FG Nup swelling due to excluded-volume interactions [Fig. 1(b)] needs to be counteracted by intramolecular cohesion. As a consequence, overestimates of protein volume, and hence of excluded volume, lead to larger $\epsilon_{p p}$ values in our comparisons with the experimental data. We note that FG Nup domains with higher charged or polar content appear more extended [5], which in our analysis translates into smaller $\epsilon_{p p}$ values. Interestingly, this further extension can be entirely explained by the larger excluded volume of these specific domains, i.e., without needing to take into account explicit electrostatic interactions (Fig. S3 of the Supplemental Material [56]). This suggests that the attractive effects of various numbers of hydrophobic, charged, and hydrophilic amino acid regions, defining the heteropolymer nature of FG Nups [5], can be suitably captured in our homopolymer model with varying values of one interaction parameter. (a)

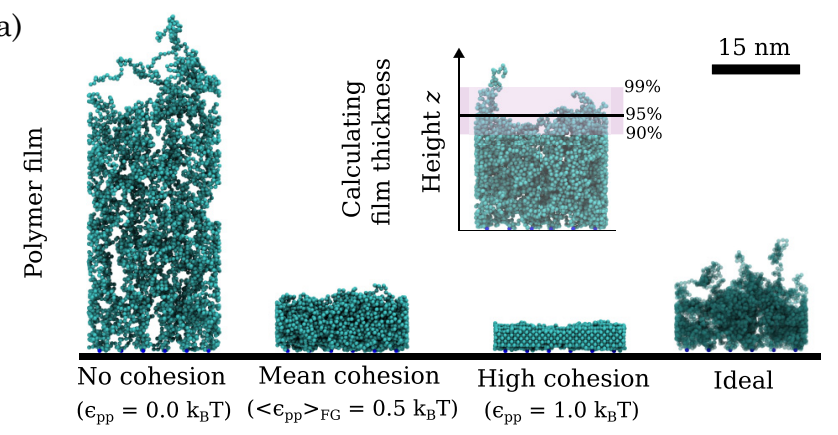

(b)

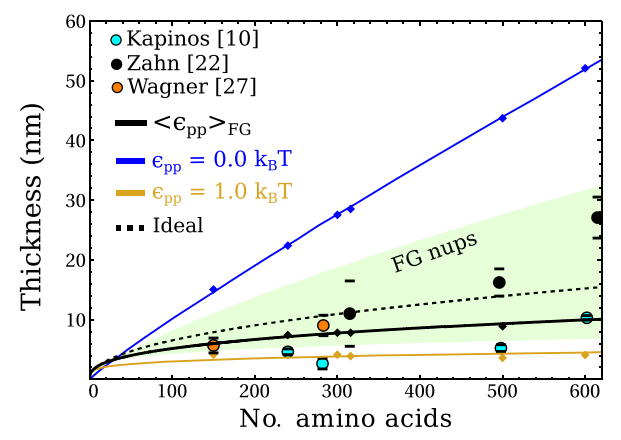

(c)

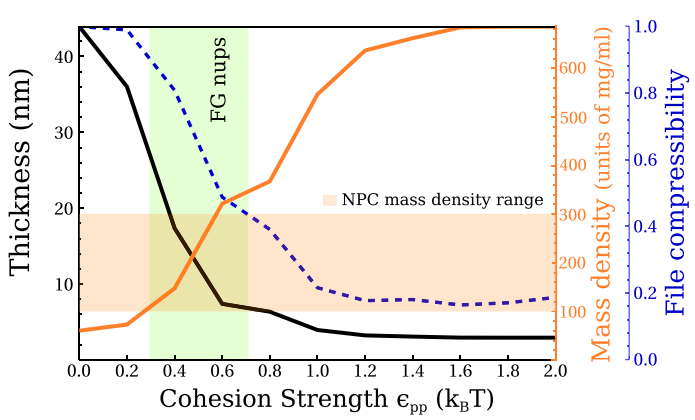

FIG. 2. Comparing the parameterized polymer model with planar assemblies of FG Nups. (a) MD snapshots of polymers ( $N=300$ beads) grafted onto a surface (at a density of 3.2 polymers $/ 100 \mathrm{~nm}^{2}$ ) for various interaction regimes. (b) Experimental film thicknesses (circles) plotted against FG Nup sequence lengths. MD simulation data (diamonds) are shown for $\epsilon_{p p}=\left\{0.0,\left\langle\epsilon_{p p}\right\rangle_{F G}, 1.0\right\} k_{B} T$ alongside ideal polymer data. (c) Measures of compaction as a function of $\epsilon_{p p}$ (MD; see main text). The mass density range, from a simulated NPC, is shown in orange shading [44].

We also tested against experimental data on FG Nups that are grafted, at physiological densities, onto a planar surface (polymer film) [10,22,27]. MD simulations (Fig. 2(a), and Video S2 of the Supplemental Material [56]) for $\left\langle\epsilon_{p p}\right\rangle_{F G}=$ $0.5 \pm 0.2 k_{B} T$ yield FG Nup film thicknesses [Fig. 2(b), green shading] that are in agreement with the experimental data (Fig. S4 of the Supplemental Material [56]), with the exception of three data points (see the Supplemental Material [56] for an explanation). With these three exceptions, experimental and computational data for $\left\langle\epsilon_{p p}\right\rangle_{F G}$ again show close agreement with the predictions for ideal polymers.

Next we explore how the identified ideal polymer morphology translates into functionally relevant properties in a polymer film assembly containing one type of FG Nup $(N=$ 300 beads). First, in the $\epsilon_{p p}$ range relevant for FG Nups, we find that the computed film thickness [Fig. 2(c)] shows 
(a)

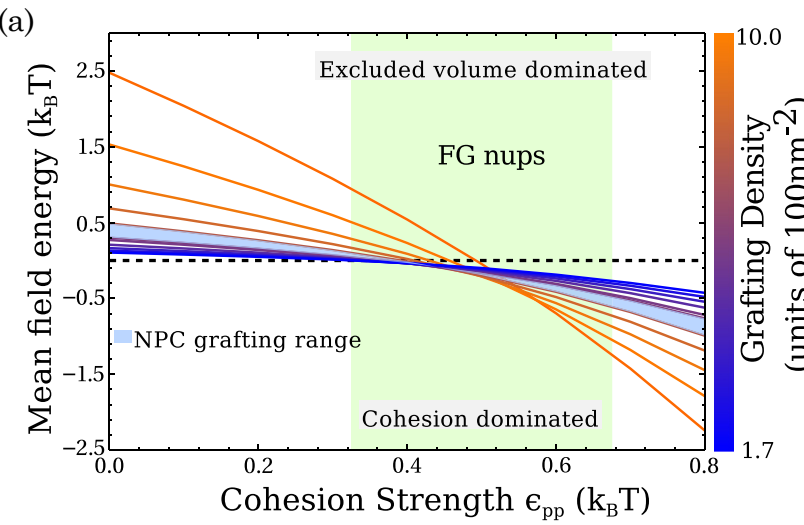

(c)

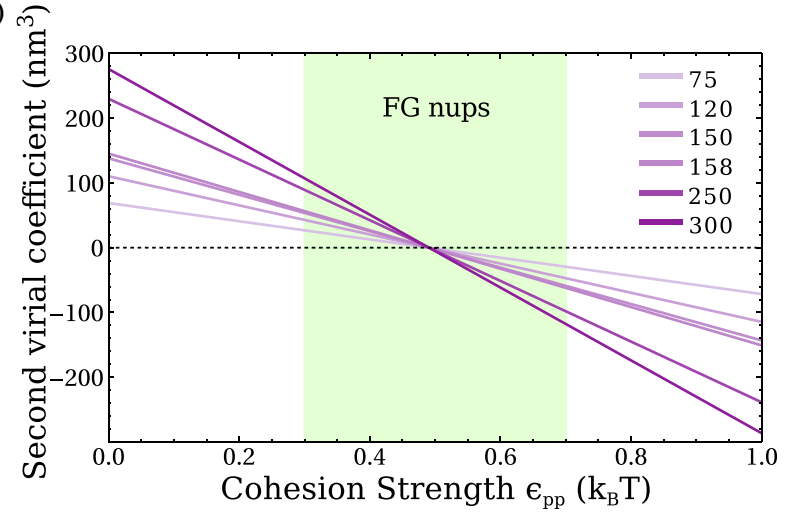

(b)

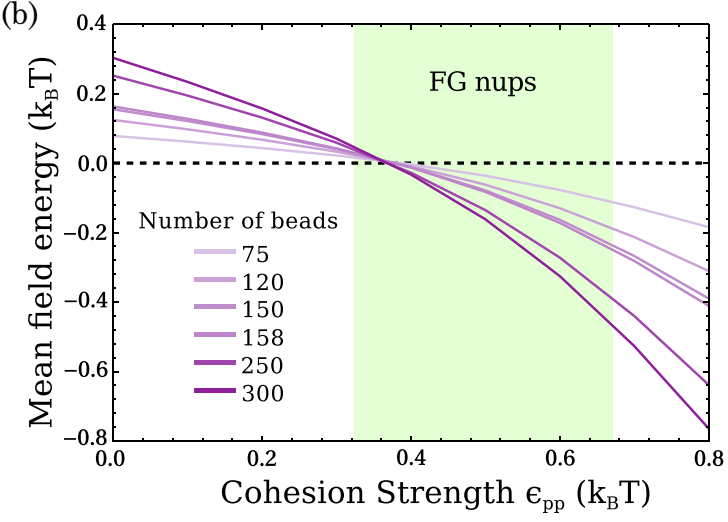

(d)

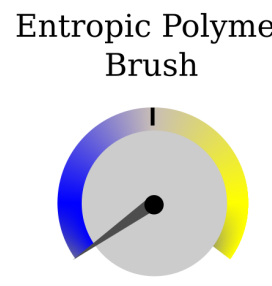

FG Nups

Cohesive

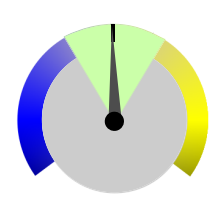

Brush

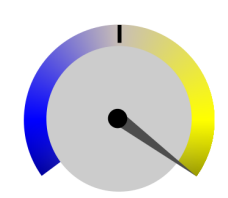

Excluded

Cohesion

volume

FIG. 3. Planar assemblies of FG Nups adopt morphologies that balance excluded-volume and cohesive interactions analyzed by DFT. (a) The mean-field energy [see Eq. (12), multiplied by $k_{B} T$ ] divided by the number of polymers as a function of $\epsilon_{p p}$ for a range of grafting densities $(N=300$ beads). (b) Same as (a), but for various polymer lengths and for a physiological grafting density of 3.2 polymers per $100 \mathrm{~nm}^{2}$. (c) The second virial coefficient per polymer (not per bead) as a function of $\epsilon_{p p}$ calculated from the bead-bead pair potential. (d) An illustration of the balance between excluded-volume and cohesive interactions for FG Nups.

a steep change from a swollen polymer film to a compact film. This is confirmed by other measures of film compaction, such as the mass density and file compressibility, where the file compressibility has been used to describe order in nonequilibrium and equilibrium many-body systems $[45,46]$. The mass density is calculated by converting the units of number density within the film thickness to $\mathrm{mg} / \mathrm{ml}$ assuming a molecular weight of $220 \mathrm{Da}$ for a polymer bead, and the file compressibility corresponds to the losslessly compressed size (in bytes), using the BZIP2 file compression library [47]), of a file containing the MD bead coordinates divided by the losslessly compressed size for $\epsilon_{p p}=0.0 k_{B} T$ (see the Supplemental Material [56]). The mass density of the FG Nups, in the relevant $\epsilon_{p p}$ range, is consistent with the FG Nup mass density in the NPC, as previously estimated using simulations which model the geometry of the nuclear pore scaffold, and the FG Nups down to the amino acid level [44].

To further elucidate the molecular interactions within the polymer film assembly, we use free energy density functional theory (DFT) modeling, in which grafted FG Nups of the same type are described as ideal polymers interacting with a mean field that is optimized to best represent the net effect of excluded-volume, cohesion, and grafting of the FG Nups to a surface [15]. This DFT yields film thicknesses that are in good agreement with the MD simulations of the same system (see Fig. S5 of the Supplemental Material [56]), and allows us to quantify the effect of molecular interactions via the mean field per polymer in the film (Fig. S6 of the Supplemental Material [56]). Here the ideal polymer behavior, where excluded volume and cohesion balance out [Fig. 3(d)], is articulated via the zero crossings of the mean-field energy per polymer and of the second virial coefficient [48] in the relevant range of $\epsilon_{p p}$ (Fig. 3).

We now investigate how the identified ideal polymer behavior translates into a pore assembly, as probed here by MD simulations on NPC mimetics based on well-defined numbers and types of FG Nups that are grafted in a DNA origami pore scaffold, with an inner radius $(\sim 20 \mathrm{~nm})$ comparable to that of the NPC [49]. We observe that the change in polymer compaction is even more abrupt (in the relevant $\epsilon_{p p}$ range) for FG Nups that are assembled in a pore geometry (Fig. 4, and Fig. S7 and Video S3 of the Supplemental Material [56]). This change in compaction results from the increased affinity between the polymers, which causes an increase in local concentration. This increased local concentration further enhances the probability of intra- and intermolecular interactions to come into play, such that attractive interactions can further compact the polymers, thus causing a highly nonlinear response to changes in $\epsilon_{p p}$. As demonstrated here, this phenomenon is particularly articulated when the majority of the polymer become confined in the nanopore confinement. Hence in the physiologically relevant parameter range as determined above, we observe that FG Nup assemblies in a 
(a)

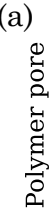

(b)

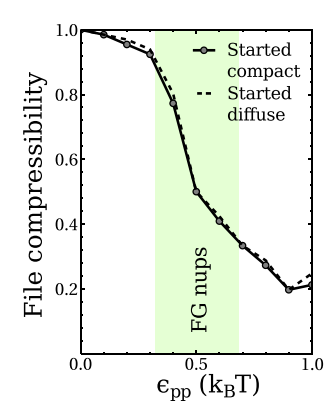

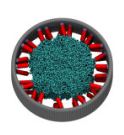

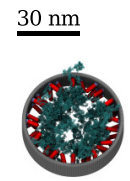

No cohesion Mean cohesion High cohesion Ideal $\left(\epsilon_{\mathrm{pp}}=0.0 \mathrm{k}_{\mathrm{B}} \mathrm{T}\right) \quad\left(<\epsilon_{\mathrm{pp}}>_{\mathrm{FG}}=0.5 \mathrm{k}_{\mathrm{B}} \mathrm{T}\right)\left(\epsilon_{\mathrm{pp}}=1.0 \mathrm{k}_{\mathrm{B}} \mathrm{T}\right)$

(c)

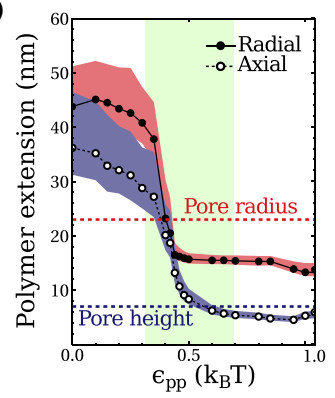

FIG. 4. Investigating

polymer

morphology

in an NPC-mimicking geometry containing one type of FG Nup [49]. (a) MD snapshots of polymers in a pore (polymers in blue; inner pore scaffold in gray; grafting handles in red) for various interaction regimes (48 polymers, $N=300$ beads). (b) File compressibility plotted as a function of $\epsilon_{p p}$. (c) Polymer extension in the pore as a function of $\epsilon_{p p}$. The red (radial) and purple (axial) bands denote the extension thresholds that contain $99 \%$ and $90 \%$ of the total beads.

nanopore can undergo major conformational changes for only minor changes in intermolecular interactions [15,50].

The polymer systems investigated thus far in this work have contained only one type of FG Nup. However, it is known that the NPC contains many different types of FG Nups with varying cohesiveness [5]. We therefore also investigated a binary polymer pore assembly, with 24 polymers with varying $\epsilon_{p p}$ and 24 noncohesive polymers, and found that the presence of the noncohesive polymers has a negligible effect on the morphology of the cohesive polymers (Fig. 5).

Thus far, we have observed that FG Nup morphologies, on average, resemble those of ideal polymers, that they are at the transition between two (extreme) regimes of polymer behavior, and, at this transition, it is possible to have large conformational changes for small changes in molecular interactions. This latter observation appears to be of significant physiological relevance, as it provides a mechanism by which FG Nup assemblies in the NPC may open and close [15] to facilitate transport of large cargos at millisecond timescales without compromising the transport barrier.

We next investigated the resealing dynamics of the barrier, i.e., how fast and by how much polymers fill the center of the pore following a perturbation: holes (void of polymers) of 10,20 , and $30 \mathrm{~nm}$ diameter are created in an NPCmimetic pore system containing 48 polymers [49] (Fig. 6(a), and Fig. S8 and Video S4 of the Supplemental Material [56]). We observe that FG Nup resealing of the pore can be characterized by two regimes depending on the cohesion strength $\epsilon_{p p}$ [Figs. 6(b) and 6(c)], where we have mapped the timestep in our MD simulations to the appropriate unit of time by comparing the diffusion of two polymer beads to that of an alanine-proline dimer [51]. For $\epsilon_{p p} \gtrsim 0.4 k_{B} T$, the
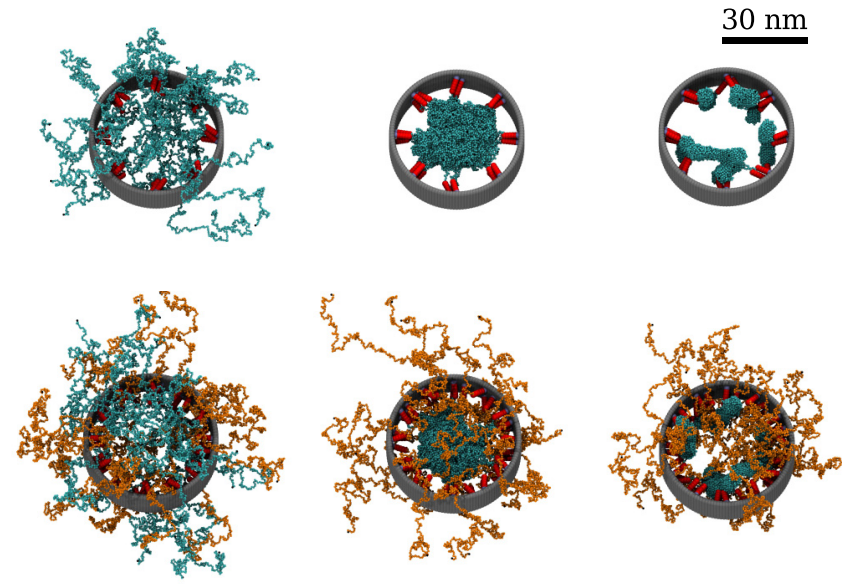

No cohesion

$$
<\epsilon_{\mathrm{pp}}>_{\mathrm{FG}}
$$

High cohesion

FIG. 5. Incorporating polymers with no cohesion, but with excluded-volume interactions still present, into a polymer pore assembly does not change the morphology of the cohesive polymers as compared with pores containing cohesive polymers only. Top: MD snapshots of cohesive polymers (blue) (24 polymers, $N=300$ beads). Bottom: MD snapshots of a binary mixture of 24 cohesive polymers (blue) and 24 noncohesive polymers (orange) $(N=300$ beads).

center of the pore reaches a maximum polymer density that is approximately double that for $\epsilon_{p p}<0.4 k_{B} T$. We also observe that for $\epsilon_{p p} \gtrsim 0.4 k_{B} T$, the resealing rate appears slower than for $\epsilon_{p p}<0.4 k_{B} T$, where polymers rapidly extend away from their tethering points (Fig. S8 of the Supplemental Material [56]). We probed the resealing time $\tau$, which is the time taken for the central density to reach $\approx 63 \%$ its equilibrium value [Fig. 6(c)]. Notably, for polymers at $\left\langle\epsilon_{p p}\right\rangle_{F G}$, the resealing time for "large cargos" (exceeding $10 \mathrm{~nm}$ diameter [1-3]) is similar to that of ideal polymers, albeit that the central density remains at a relatively lower level for the ideal polymer case. This implies that the similarity to ideal polymers also extends to the dynamic behavior of FG Nups. Typically, the resealing times are on the microsecond timescale, much faster-as needed to inhibit unspecific transport-than the millisecond timescale for transport events, and in agreement with previous simulations [52]. We also observe a sharp transition in the resealing time occurring at $0.3<\epsilon_{p p}<0.4 k_{B} T$, which falls in the relevant $\epsilon_{p p}$ range where we observed a drastic change in polymer structure [Figs. 4(b) and 4(c)] and the ideal polymer behavior [Figs. 1(c) and 2(b)], while interestingly it is not obvious what causes this observation.

Overall we can see that in the physiologically relevant range, a small change in the cohesiveness can drastically change the polymer morphology, which would contribute to the ease of the opening and closing of the pore. However, the timescale of the polymer dynamics is not greatly affected by changes in the cohesiveness $\left(\leqslant 1.0 k_{B} T\right)$, assuring fast resealing and rapid transportation of molecules.

Finally, we investigate how the cohesive interactions between FG Nups affect dynamics at molecular and submolecular length scales. Nuclear magnetic resonance spectroscopy indicates fast (on the picosecond to nanosecond timescale) FG Nup residue dynamics [53,54], presumably indicative of entropically dominated FG Nup behavior. We simulated isolated 
(a)

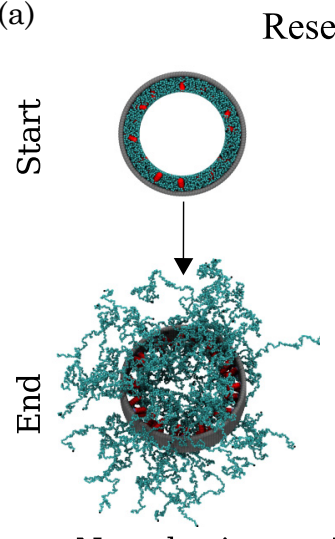

No cohesion

(c)

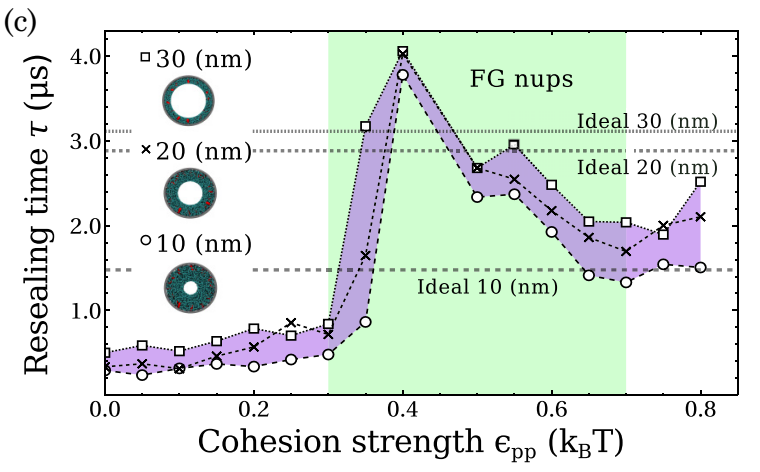

(b)
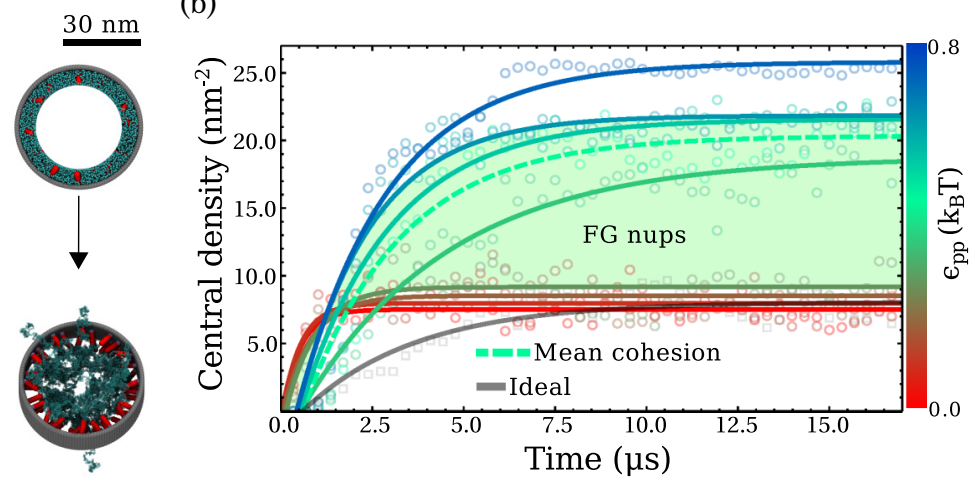

(d)

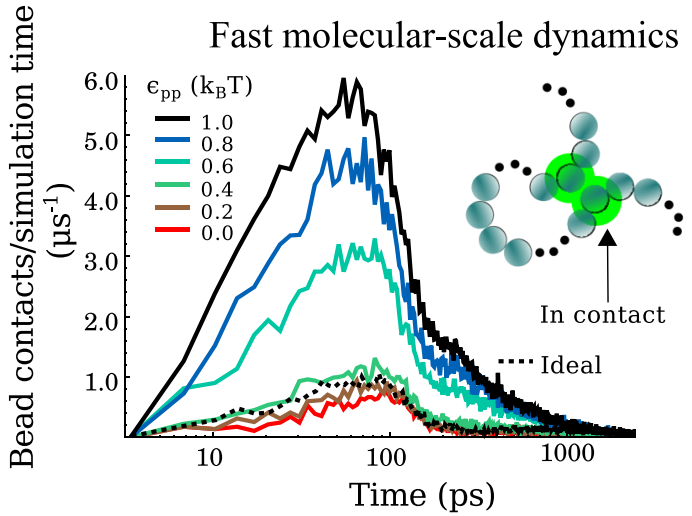

FIG. 6. FG Nup dynamics on the mesoscale and molecular scale. (a) In silico polymer resealing in a nanopore [49]. The arrow represents $34 \mu \mathrm{s}$ (or $10^{7}$ time steps) of simulation time, pointing to resulting MD snapshots. (b) The number of beads in a central circular cross-sectional area $(5 \mathrm{~nm}$ radius $)$ as a function of time, where the pore begins with a hole, $30 \mathrm{~nm}$ in diameter, at its center. The cohesion strength color bar represents the range $\epsilon_{p p}=0.0-0.8 k_{B} T$ (with $0.1 k_{B} T$ increments). Lines represent fits using $\rho(t)=\rho_{\max }[1-\exp (-t / \tau)]$, where $\rho_{\max }$ is the maximum central density and $\tau$ is the resealing time $(N=300)$. (c) $\tau$ as a function of $\epsilon_{p p}$ alongside resealing times for ideal polymers (dashed). (d) Distribution of contact durations for a single isolated polymer ( $N=300$ beads).

polymers and measured the durations of bead-bead contacts, i.e., the elapsed time during which the centers of adjacent beads (excluding nearest bonded neighbors) are within the range of the attractive pair potential [Fig. 6(d)]. Of note, the relevant timescales appear rather independent of the molecular interactions, which is due to the weak $\left(\epsilon_{p p} \leqslant 1.0 k_{B} T\right)$ interaction strengths explored here. The strong dependence of the collective FG Nup morphology on $\epsilon_{p p}$ (Figs. 1 and 2) is due to the rapid accumulation of many weak individual beadbead contacts upon a moderate increase of $\epsilon_{p p}$, which can be attributed to the higher local bead concentration for larger $\epsilon_{p p}$. We observe that the overall dynamics does not depend on the exact shape of the model potential (Fig. S9 of the Supplemental Material [56]). Overall, individual FG Nups exhibit fast dynamics (picosecond timescale) at (sub-)molecular length scales, characteristic of their entropic nature. At larger length scales, FG Nup assemblies, dominated by the collective FG Nup molecular interactions, exhibit microsecond dynamics with enhanced resealing (due to cohesion) which is of sufficient speed to maintain the transport barrier.

\section{CONCLUSION}

In summary, we find that FG Nup morphologies agree with neither predictions for purely entropic behavior, dominated by (repulsive) excluded-volume interactions, nor with predictions for gel-like, strongly condensed behavior governed by (attractive) cohesive interactions. This is fully consistent with previous studies [16,21,22,24]. More surprisingly, we find that on average, FG Nup morphologies are consistent with the predictions for polymers that are characterized by the balance of repulsive and cohesive molecular interactions, i.e., the FG Nups, on average, behave like ideal polymers, at or close to their $\Theta$-point in physiological buffer solutions. FG Nup mesoscale dynamics (microseconds) are also in agreement with predictions for ideal polymers, and are such that FG Nup assemblies can reseal fast enough to reseal the barrier after transport events, which occur on millisecond timescales. Additionally, the rapid movement of FG Nups on the molecular scale-maintained through a large range of cohesion strengths - will facilitate the uptake and release of nuclear transport factors and associated cargos [55], whereas the accumulation of many weakly cohesive interactions facilitates the tight sealing of the NPC transport barrier. Taken together, this physical picture reconciles previous-apparently contradictory-identifications of FG Nup assemblies as "entropic brushes" or "gels," and provides a conceptual framework with which to interpret the transport selectivity of the NPC (see Supplemental Material [56]). 


\section{ACKNOWLEDGMENTS}

We thank Dino Osmanović (MIT), Roy Beck (Tel-Aviv), Larissa Kapinos (Basel), Roderick Lim (Basel), Ralf Richter
(Leeds), and Anton Zilman (Toronto) for discussions. This work was funded by the Royal Society (A.Š.) and the UK EPSRC (Grant No. EP/L504889/1; L.K.D.).
[1] T. Jovanovic-Talisman and A. Zilman, Protein Transport by the Nuclear Pore Complex: Simple Biophysics of a Complex Biomachine, Biophys. J. 113, 6 (2017).

[2] I. V. Aramburu and E. A. Lemke, Floppy but not sloppy: Interaction mechanism of FG-nucleoporins and nuclear transport receptors, Semin. Cell Dev. Biol. 68, 34 (2017).

[3] G. J. Stanley, A. Fassati, and B. W. Hoogenboom, Biomechanics of the transport barrier in the nuclear pore complex, Semin. Cell Dev. Biol. 68, 42 (2017).

[4] B. B. Hülsmann, A. A. Labokha, and D. Görlich, The permeability of reconstituted nuclear pores provides direct evidence for the selective phase model, Cell 150, 738 (2012).

[5] J. Yamada, J. L. Phillips, S. Patel, G. Goldfien, A. CalestagneMorelli, H. Huang, R. Reza, J. Acheson, V. V. Krishnan, S. Newsam, A. Gopinathan, E. Y. Lau, M. E. Colvin, V. N. Uversky, and M. F. Rexach, A Bimodal Distribution of Two Distinct Categories of Intrinsically Disordered Structures with Separate Functions in FG Nucleoporins, Mol. Cell. Proteomics 9, 2205 (2010).

[6] M. Peyro, M. Soheilypour, B. Lee, and M. Mofrad, Evolutionarily conserved sequence features regulate the formation of the FG network at the center of the nuclear pore complex, Sci. Rep. 5, 15795 (2015).

[7] D. P. Denning, S. S. Patel, V. Uversky, A. L. Fink, and M. Rexach, Disorder in the nuclear pore complex: The FG repeat regions of nucleoporins are natively unfolded, Proc. Natl. Acad. Sci. USA 100, 2450 (2003).

[8] R. Y. Lim, B. Fahrenkrog, J. Köser, K. Schwarz-Herion, J. Deng, and U. Aebi, Nanomechanical basis of selective gating by the nuclear pore complex, Science 318, 640 (2007).

[9] R. L. Schoch, L. E. Kapinos, and R. Y. H. Lim, Nuclear transport receptor binding avidity triggers a self-healing collapse transition in FG-nucleoporin molecular brushes, Proc. Natl. Acad. Sci. USA 109, 16911 (2012).

[10] L. E. Kapinos, R. L. Schoch, R. S. Wagner, K. D. Schleicher, and R. Y. H. Lim, Karyopherin-centric control of nuclear pores based on molecular occupancy and kinetic analysis of multivalent binding with FG nucleoporins, Biophys. J. 106, 1751 (2014).

[11] B. L. Timney, B. Raveh, R. Mironska, J. M. Trivedi, S. J. Kim, D. Russel, S. R. Wente, A. Sali, and M. P. Rout, Simple rules for passive diffusion through the nuclear pore complex, J. Cell Biol. 215, 57 (2016).

[12] A. A. Labokha, S. Gradmann, S. Frey, B. B. Hülsmann, H. Urlaub, M. Baldus, and D. Görlich, Systematic analysis of barrier-forming FG hydrogels from Xenopus nuclear pore complexes, EMBO J. 32, 204 (2013).

[13] H. B. Schmidt and D. Görlich, Nup98 FG domains from diverse species spontaneously phase-separate into particles with nuclear pore-like permselectivity, Elife 4, e04251 (2015).

[14] O. Peleg, M. Tagliazucchi, M. Kröger, Y. Rabin, and I. Szleifer, Morphology control of hairy nanopores, ACS Nano 5, 4737 (2011).
[15] D. Osmanovic, J. Bailey, A. H. Harker, A. Fassati, B. W. Hoogenboom, and I. J. Ford, Bistable collective behavior of polymers tethered in a nanopore, Phys. Rev. E 85, 061917 (2012).

[16] N. B. Eisele, A. A. Labokha, S. Frey, D. Görlich, and R. P. Richter, Cohesiveness tunes assembly and morphology of FG nucleoporin domain meshworks - Implications for nuclear pore permeability, Biophys. J. 105, 1860 (2013).

[17] D. Osmanovic and Y. Rabin, Effect of grafting on aggregation of intrinsically disordered proteins, Biophys. J. 114, 534 (2018).

[18] M. Tagliazucchi, O. Peleg, M. Kröger, Y. Rabin, and I. Szleifer, Effect of charge, hydrophobicity, and sequence of nucleoporins on the translocation of model particles through the nuclear pore complex, Proc. Natl. Acad. Sci. USA 110, 3363 (2013).

[19] R. Gamini, W. Han, J. E. Stone, and K. Schulten, Assembly of Nsp1 Nucleoporins Provides Insight into Nuclear Pore Complex Gating, PLoS Comput. Biol. 10, e1003488 (2014).

[20] A. Ghavami, E. Van der Giessen, and P. R. Onck, Sol-gel transition in solutions of FG-Nups of the nuclear pore complex, Extrem. Mech. Lett. 22, 36 (2018).

[21] A. Vovk, C. Gu, M. G. Opferman, L. E. Kapinos, R. Y. H. Lim, R. D. Coalson, D. Jasnow, and A. Zilman, Simple biophysics underpins collective conformations of the intrinsically disordered proteins of the Nuclear Pore Complex, Elife 5, e10785 (2016).

[22] R. Zahn, S. Ehret, C. A. Callis, D. Osmanovic, S. Frey, M. Stewart, C. You, D. Gorlich, R. P. Richter, and B. W. Hoogenboom, A physical model describing the interaction of nuclear transport receptors with FG nucleoporin domain assemblies, Elife 5, e14119 (2016).

[23] H. Hofmann, A. Soranno, A. Borgia, K. Gast, D. Nettels, and B. Schuler, Polymer scaling laws of unfolded and intrinsically disordered proteins quantified with single-molecule spectroscopy, Proc. Natl. Acad. Sci. USA 109, 16155 (2012).

[24] G. Fuertes, N. Banterle, K. M. Ruff, A. Chowdhury, D. Mercadante, C. Koehler, M. Kachala, G. Estrada Girona, S. Milles, A. Mishra, P. R. Onck, F. Gräter, S. Esteban-Martín, R. V. Pappu, D. I. Svergun, and E. A. Lemke, Decoupling of size and shape fluctuations in heteropolymeric sequences reconciles discrepancies in SAXS vs FRET measurements, Proc. Natl. Acad. Sci. USA 114, E6342 (2017).

[25] P.-G. de. Gennes, Scaling Concepts in Polymer Physics (Cornell University Press, Ithaca, NY, 1979).

[26] D. P. Denning, V. Uversky, S. S. Patel, A. L. Fink, and M. Rexach, The Saccharomyces cerevisiae nucleoporin Nup2p is a natively unfolded protein, J. Biol. Chem. 277, 33447 (2002).

[27] R. S. Wagner, L. E. Kapinos, N. J. Marshall, M. Stewart, and R. Y. H. Lim, Promiscuous binding of karyopherin $\beta 1$ modulates FG nucleoporin barrier function and expedites NTF2 transport kinetics, Biophys. J. 108, 918 (2015).

[28] D. Osmanović, I. J. Ford, and B. W. Hoogenboom, Model inspired by nuclear pore complex suggests possible roles for 
nuclear transport receptors in determining its structure, Biophys. J. 105, 2781 (2013).

[29] R. Y. H. Lim, N.-P. Huang, J. Koser, J. Deng, K. H. A. Lau, K. Schwarz-Herion, B. Fahrenkrog, and U. Aebi, Flexible phenylalanine-glycine nucleoporins as entropic barriers to nucleocytoplasmic transport, Proc. Natl. Acad. Sci. USA 103, 9512 (2006).

[30] G. Stirnemann, D. Giganti, J. M. Fernandez, and B. J. Berne, Elasticity, structure, and relaxation of extended proteins under force, Proc. Natl. Acad. Sci. USA 110, 3847 (2013).

[31] R. Simpson, Proteins and Proteomics: A Laboratory Manual (Cold Spring Harbour Laboratory Press, New York, 2004).

[32] J. N. Israelachvili, Intermolecular Surface Forces, 3rd ed. (Academic Press, New York, 2011), pp. 1-676.

[33] M. P. Allen and D. J. Tildesley, Computer Simulation of Liquids (Oxford University Press, Oxford, 1987).

[34] S. Plimpton, Fast Parallel Algorithms for Short-Range Molecular Dynamics, J. Comput. Phys. 117, 1 (1995).

[35] R. Evans, The nature of the liquid-vapour interface and other topics in the statistical mechanics of non-uniform, classical fluids, Adv. Phys. 28, 143 (1979).

[36] R. Roth, R. Evans, A. Lang, and G. Kahl, Fundamental measure theory for hard-sphere mixtures revisited: The white bear version, J. Phys. Condens. Matter 14, 12063 (2002).

[37] Y. X. Yu and J. Wu, Density functional theory for inhomogeneous mixtures of polymeric fluids, J. Chem. Phys. 117, 2368 (2002).

[38] R. Roth, Fundamental measure theory for hard-sphere mixtures: A review, J. Phys. Condens. Matter 22, 063102 (2010).

[39] A. J. Archer, B. Chacko, and R. Evans, The standard meanfield treatment of interparticle attraction in classical DFT is better than one might expect, J. Chem. Phys. 147, 034501 (2017).

[40] D. H. D. Jong, L. V. Schäfer, A. H. D. Vries, S. J. Marrink, H. J. C. Berendsen, and H. Grubmüller, Determining equilibrium constants for dimerization reactions from molecular dynamics simulations, J. Comput. Chem. 32, 1919 (2011).

[41] A. Ghavami, E. van der Giessen, and P. R. Onck, Coarsegrained potentials for local interactions in unfolded proteins, $\mathrm{J}$. Chem. Theory Comput. 9, 432 (2012).

[42] J. G. De La Torre, G. Del Rio Echenique, and A. Ortega, Improved calculation of rotational diffusion and intrinsic viscosity of bead models for macromolecules and nanoparticles, J. Phys. Chem. B 111, 955 (2007).

[43] A. J. Ladd and D. Frenkel, Computer simulation studies of static and dynamical scaling in dilute solutions of excluded-volume polymers, Macromolecules 25, 3435 (1992).

[44] A. Ghavami, L. M. Veenhoff, E. Van Der Giessen, and P. R. Onck, Probing the disordered domain of the nuclear pore com- plex through coarse-grained molecular dynamics simulations, Biophys. J. 107, 1393 (2014).

[45] R. Avinery, M. Kornreich, and R. Beck, Universal and Efficient Entropy Estimation Using a Compression Algorithm, Phys. Rev. Lett. 123, 178102 (2019).

[46] S. Martiniani, R. Alfia, P. M. Chaikin, and D. Levine, Quantifying Hidden Order Out of Equilibrium, Phys. Rev. X 9, 011031 (2017).

[47] J. Seward, Bzip2 and libbzip2: Version 1.0.5, A program and library for data compression, 2007, http://www.bzip.org/ (unpublished).

[48] G. Strobl, The Physics of Polymers: Concepts for Understanding Their Structures and Behavior (Springer, Berlin, 2007).

[49] P. D. E. Fisher, Q. Shen, B. Akpinar, L. k. Davis, K. K. H. Chung, D. Baddeley, A. Saric, T. J. Melia, B. W. Hoogenboom, C. Lin, and C. P. Lusk, A programmable DNA origami platform for organizing intrinsically disordered nucleoporins within nanopore confinement, ACS Nano 12, 1508 (2018).

[50] S. Ro, A. Gopinathan, and Y. W. Kim, Interactions between a fluctuating polymer barrier and transport factors together with enzyme action are sufficient for selective and rapid transport through the nuclear pore complex, Phys. Rev. E 98, 012403 (2018).

[51] P. Mark and L. Nilsson, Molecular dynamics simulations of the Ala-Pro dipeptide in water: Conformational dynamics of trans and cis isomers using different water models, J. Phys. Chem. B 105, 8028 (2001).

[52] R. Moussavi-Baygi and M. R. Mofrad, Rapid brownian motion primes ultrafast reconstruction of intrinsically disordered phegly repeats inside the nuclear pore complex, Sci. Rep. 6, 29991 (2016).

[53] L. E. Hough, K. Dutta, S. Sparks, D. B. Temel, A. Kamal, J. Tetenbaum-Novatt, M. P. Rout, and D. Cowburn, The molecular mechanism of nuclear transport revealed by atomic-scale measurements, Elife 4, e10027 (2015).

[54] S. Milles, D. Mercadante, I. V. Aramburu, M. R. Jensen, N. Banterle, C. Koehler, S. Tyagi, J. Clarke, S. L. Shammas, M. Blackledge, F. Gräter, and E. A. Lemke, Plasticity of an ultrafast interaction between nucleoporins and nuclear transport receptors, Cell 163, 734 (2015).

[55] R. Hayama, S. Sparks, L. M. Hecht, K. Dutta, J. M. Karp, C. M. Cabana, M. P. Rout, D. Cowburn, and N. M. Allewell, Thermodynamic characterization of the multivalent interactions underlying rapid and selective translocation through the nuclear pore complex, J. Biol. Chem. 293, 4555 (2018).

[56] See Supplemental Material at http://link.aps.org/supplemental/ 10.1103/PhysRevE.101.022420 for explicit descriptions of the simulation and data analysis methods employed throughout this manuscript, and for the supplemental figures and supplemental video legends. 